\title{
Extended Cavity Model to Analysis Tunable Circular Disk Microstrip Antenna Using Genetic Algorithm
}

\author{
Sami BEDRA ${ }^{1}$, Tarek FORTAKI ${ }^{2}$, Siham BENKOUDA ${ }^{3}$, Abderraouf MESSAI ${ }^{3}$ \\ ${ }^{1}$ Industrial Engineering Department, University of Khenchela, 40004 Khenchela, Algeria \\ ${ }^{2}$ Electronics Department, University of Batna, 05000 Batna, Algeria \\ ${ }^{3}$ Electronics Department, University of Constantine1, 25000 Constantine, Algeria \\ bedra_sami@yahoo.fr
}

\begin{abstract}
In this paper, the cavity model for simple circular disc microstrip antenna is extended with some modifications for the tunable geometry taking into account the anisotropy in the layer. The numerical results show that there are substantial deviations in calculated resonant frequency when substrate dielectric anisotropy is considered. Furthermore, significant variations are seen in the radiation patterns of the structures due to substrate anisotropy. Finally the effect of inclusion of air gap layer inserted between substrate and ground plane on the resonant characteristics is also investigated for fundamental and higher order modes.
\end{abstract}

Keywords—cavity model; genetic algorithm; anisotropic substrate; adjustable air gap.

\section{I.Introduction}

Microstrip antennas are becoming increasingly popular since they have small volume and a low-profile planar configuration. Easy mass production of such antennas using printed circuit technology leads to low fabrication cost. They are much easier to be integrated into microwave circuits on the same substrate. Especially, they can be made conformal to the host surface [1].

Some dielectric substances exhibit anisotropy due to their natural crystal structures or as the result of their production processes. Isotropic substances may also exhibit anisotropy at high frequencies. In the design of microwave integrated circuit components and microstrip antennas, anisotropic substances have been increasingly popular [2-10]. Uniaxial substrates have drawn more attention due to their availability such as sapphire, boron nitride and E-10 ceramic-impregnated Teflon. Their main drawback is narrow bandwidth characteristics, which is considerably avoided by operating the antenna around the resonant frequency. As an alternative, doublelayered structure with air gap having adjustable thickness between the substrate and the ground plane is also found to be useful in obtaining the wide band operation. For both single and double-layered structures, accurate computation

of resonant frequency is an important task and takes considerable interest in literature by various authors

depending on the usage of various methods and approximations [11-19]. In this study, resonant frequency of double layered circular patch microstrip antenna is accurately determined via cavity analysis, using a simple effective permittivity and patch radius expressions including modal effects. The aim of this work to perform an accurate and efficient analysis of circular-disc microstrip antennas on double layer, as well as to perform the analyses for circular microstrip antennas on a single layer substrate and on a tunable substrate, as particular cases.

\section{II.Antenna Configuration and Design}

The tunable circular microstrip antenna structure is shown in Fig.1. The resonant behavior of the antenna is independent of the feed so that the feed was not taken into account in the analysis.

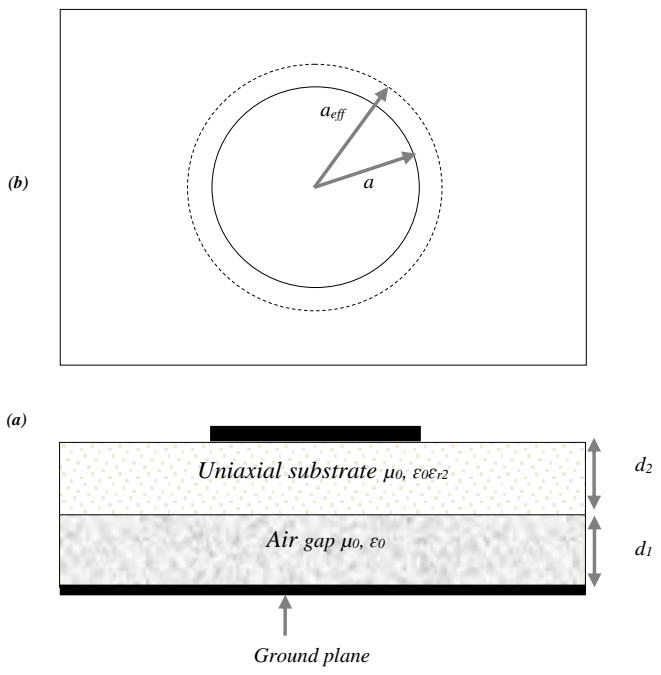

Fig. 1. Geometry of a circular microstrip antenna with air gap.

a- Side view

b- Top view 
doi:10.15849/icit.2015.0117 @ ICIT 2015 (http://icit.zuj.edu.jo/ICIT15)

The Resonant frequency of this antenna can be determined from cavity model for various operational modes and structural parameters using proper equivalent model with effective structural parameters [3]. For this purpose, various expressions for effective patch radius $a_{\text {eff }}$ and effective relative permittivity $\varepsilon_{\text {eff }}$ are defined in literature $[13,15,18$, 19].

In this study, effective patch radius expression to approximate the modal effects is taken for the double-layered antenna in the modified form:

$$
f_{r}=\frac{\chi_{n m} v_{0}}{2 \pi a \sqrt{\varepsilon_{r e q}}}
$$

where $\chi_{\mathrm{nm}}$ is the mth zero of the derivative of the Bessel function of order $\mathrm{n}$, the value of which $\left(\chi_{01}=3.832, \chi_{11}=1.841\right.$, $\left.\chi_{21}=3.054, \chi_{31}=4.201\right)$ determines the lowest and higher order modes as $\mathrm{TM}_{11}, \mathrm{TM}_{21}, \mathrm{TM}_{01}$, and $\mathrm{TM}_{31}$ modes.

$v_{0}$ is the velocity of light in free space, $a$ is the patch radius, and $\varepsilon_{r e q}$ is the substrate relative permittivity of the equivalent structure which can be determined from the cavity model [14]

$$
\varepsilon_{r e q}=\varepsilon_{r 2}\left(d_{1}+d_{2}\right) /\left(\varepsilon_{r 2} d_{1}+d_{2}\right)
$$

To account for the fact that small fraction of the field exists outside the dielectric; it is customary to use effective permittivity $\varepsilon_{\text {eff }}$ in place of $\varepsilon_{\text {req }}$

$$
\varepsilon_{e f f}=\varepsilon_{r e q}-0.9 \varepsilon_{r e q}\left[\frac{2 d}{a}+\left(\frac{d}{a}\right)^{2}\right]
$$

Where $d=d_{1}+d_{2}$ and, $\varepsilon_{r 2}$ is the relative permittivity of dielectric substrate.

If we want to take the substrate uniaxial anisotropy's into account, the relative dielectric permittivity $\varepsilon_{r}$ will be replaced with the tensor $\varepsilon_{r}=\operatorname{diag}\left(\varepsilon_{x}, \varepsilon_{x}, \varepsilon_{z}\right)$ where $\varepsilon_{x}$ and $\varepsilon_{z}$ are the relative dielectric permittivity along $\mathrm{x}$ and $\mathrm{z}$ axis, respectively

$\circ$ For the case of isotropic substrate with air gap, we use the effective dielectric constant $\varepsilon_{e q}$ given in Eq. (2).

- For the case of uniaxially anisotropic substrate without air gap, $\varepsilon_{e q}$ given in [21] Eq. (2) is used to determine de, there resulting values are:

$$
\varepsilon_{r e q}=\varepsilon_{z}
$$

$$
d_{e}=d \sqrt{\frac{\varepsilon_{x}}{\varepsilon_{z}}}
$$

To account fringe field effects, the circular patch radius a given in Eq. (1) should be replaced by its effective value [20,21]. In this letter, a new effective patch radius expression is presented to compute the resonant frequency of a circular MSA with thin and without air gap for providing better accuracy. By utilizing the experimental data reported elsewhere [22-27], after many trials, the following model, depending on $\varepsilon_{\text {eff }}, a$ and $d$, which produces good results, was chosen

$$
a_{\text {eff }}=a+\left[\beta_{1}+\left(\frac{\beta_{2}}{\varepsilon_{\text {eff }} \beta_{3}}\right)\right] d+\left(\frac{\beta_{4}}{a}\right) d^{2}
$$

where the unknown coefficients are determined by a genetic optimization algorithm. It is evident from (6) that the effective patch radius, $a_{\text {eff }}$ is larger than the physical patch radius, $a$, provided the conditions and are satisfied. In the following section, the genetic optimization algorithm used in this work is described and then the application of the genetic algorithm to the problem is explained.

\section{III.Genetic Algorithm}

The GA $[28,29]$ is based on the evolution theory where weak species face extinction but strong ones survive and pass their genes to the next generation. However for the strong species to survive there is also a requirement for random injection of genes. As GA mainly manipulates matrices it is normally implemented using Matlab software. The step by step procedure of generating the software program is shown below.

Step 1: Each variable is assigned a number of binary digits so that the required accuracy of this variable is obtained in the final solution.

Step 2: All the variables in their binary form are grouped into a string which is called a chromosome.

Step 3: Matlab is used to select a fixed number of random chromosomes called a population out of all possible number of chromosomes that are present. This is called the current generation.

Step 4: Converting the digital value of each variable in a chromosome to an analogue value, the objective function $(\mathrm{F})$ is evaluated and the relative fitness of each chromosome (Pi) determined. This relative fitness is defined as:

$$
F=\sum_{i=1}^{n} \operatorname{eval}_{i}\left[P_{i}\right]
$$

Step 5: The selective probability is determined by: 
ICIT 2015 The $7^{\text {th }}$ International Conference on Information Technology

doi:10.15849/icit.2015.0117 (C) ICIT 2015 (http://icit.zuj.edu.jo/ICIT15)

$$
P_{s i}=\frac{\operatorname{eval}_{i}\left[P_{i}\right]}{F}
$$

The cumulative probability of the chromosomes is given as:

$$
q_{i}=\sum_{j=1}^{n} P_{s j}
$$

Then a random number ' $r$ ' is generated in the range 0 to 1 . If qi- $1 \leq \mathrm{r} \leq$ qi then select Psi.

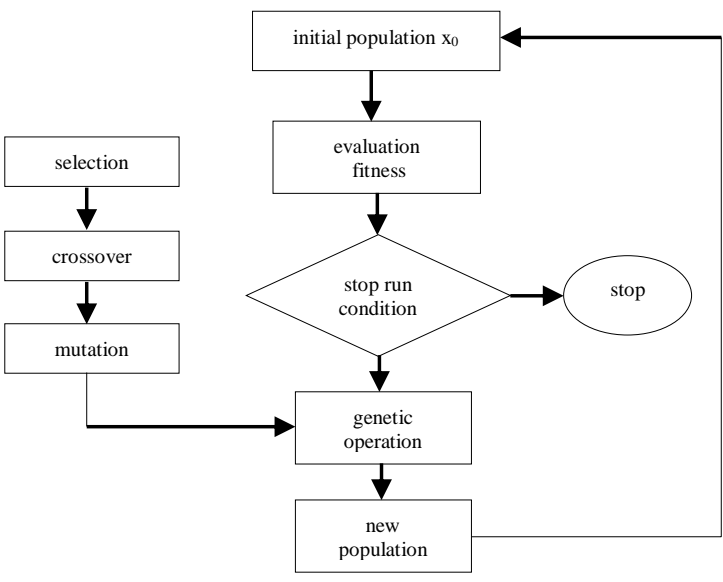

Fig. 2. Flow chart of genetic algorithm.

Step 6: Crossover is applied for random chromosomes between the parent and next generation to produce new off springs.

Step 7: The population is mutated by changing in a random way the value of the genes with the least significant bit having the highest probability of mutation and the most significant the least. The flowchart of GA is shown in Figure 2.

The next generation now becomes the parent generation and the above process is repeated until the genetic variation in the population is below a certain threshold.

As the number of generations increases both the cross over rate and the mutation rate are gradually reduced.

where $\beta_{1}, \beta_{2}, \beta_{3}$ and $\beta_{4}$ are given in the above equation are the coefficients to be determined by GA so as to minimize the following total absolute errors (TAE)

$$
T A E=\sum\left|f_{m e}-f_{c a}\right|
$$

where fme and fca are, respectively, the measured and calculated resonant frequency of circular MSA.

The control parameters in the optimization are as follows:

Maximum number of generations: 50

Population size: 100

Probability of 0 in initial population: 0.15

Probability of crossover: 0.8

Probability of mutation: 0.05

Optimization object: resonant frequency

\begin{tabular}{|c|c|c|c|c|c|c|c|c|}
\hline \multicolumn{3}{|c|}{ Physical and Electrical Parameters } & \multirow{2}{*}{$\begin{array}{c}\text { Measured } \\
f r \\
(\boldsymbol{G H} z)\end{array}$} & \multicolumn{3}{|c|}{ Calculated Frequencies fr $(\mathrm{GHz})$} & \multirow{2}{*}{$\begin{array}{c}\text { Our results } \\
\begin{array}{c}(\mathbf{G H z}) \\
f_{r}\end{array}\end{array}$} & \multirow{2}{*}{$\begin{array}{c}\text { Measured } \\
\text { By }\end{array}$} \\
\hline$d(m m)$ & $\varepsilon_{r 2}$ & $a(m m)$ & & [23] & [26] & [27] & & \\
\hline 1.588 & 2.5 & 34.93 & 1.57 & 1.592 & 1.555 & 1.559 & 1.557 & \multirow{2}{*}{ [23] } \\
\hline 3.175 & 2.5 & 34.93 & 1.51 & 1.592 & 1.522 & 1.529 & 1.522 & \\
\hline 2.35 & 4.55 & 49.5 & 0.825 & 0.832 & 0.827 & 0.827 & 0.824 & \multirow{5}{*}[26]{} \\
\hline 2.35 & 4.55 & 29.9 & 1.36 & 1.378 & 1.358 & 1.360 & 1.355 & \\
\hline 2.35 & 4.55 & 20 & 2.003 & 2.060 & 2.009 & 2.012 & 2.007 & \\
\hline 2.35 & 4.55 & 10.4 & 3.75 & 3.962 & 3.743 & 3.737 & 3.750 & \\
\hline 2.35 & 4.55 & 7.7 & 4.945 & 5.352 & 4.938 & 4.924 & 4.947 & \\
\hline 1.5875 & 2.65 & 11.5 & 4.425 & 4.695 & 4.414 & 4.438 & 4.416 & \multirow{3}{*}[22]{} \\
\hline 1.5875 & 2.65 & 10.7 & 4.723 & 5.046 & 4.724 & 4.750 & 4.723 & \\
\hline 1.5875 & 2.65 & 8.2 & 6.074 & 7.297 & 6.049 & 6.086 & 6.034 & \\
\hline
\end{tabular}

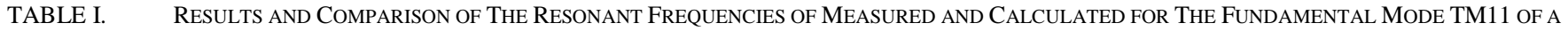
CIRCULAR ANTENNA AND THE NO GAP CASE.

TABLE II. COMPARISON OF THE RESONANT FREQUENCIES OF MEASURED AND CALCULATED OF A CIRCULAR ANTENNA HAVING AN AIR GAP: $\mathrm{a}=50 \mathrm{~mm}, \varepsilon_{r}=2.32, \mathrm{~d}_{2}=1.59 \mathrm{~mm}$.

\begin{tabular}{|c|c|c|c|c|c|c|c|}
\hline \multirow{3}{*}{ Mode $\mathbf{T M}_{\mathbf{n m}}$} & \multirow{2}{*}{$\mathbf{d}_{\mathbf{1}}(\mathbf{m m})$} & $\begin{array}{c}\text { Measured } \\
\boldsymbol{f}_{\boldsymbol{r}}(\mathbf{G H z})\end{array}$ & \multicolumn{4}{|c|}{ Calculated Frequencies $\boldsymbol{f}_{\boldsymbol{r}}(\mathbf{G H z})$} & \multirow{2}{*}{$\begin{array}{c}\text { Our results }(\mathbf{G H z}) \\
\boldsymbol{f}_{\boldsymbol{r}}\end{array}$} \\
\cline { 3 - 7 } & & Dahele [12] & Abboud [13] & Gurel [16] & Guha [17] & HFSS [31] & 1.134 \\
\hline \multirow{3}{*}{$\mathrm{TM}_{11}$} & 0 & 1.128 & 1.159 & 1.129 & 1.130 & 1.162 & 1.283 \\
\cline { 2 - 7 } & 0.5 & 1.286 & 1.298 & 1.281 & 1.274 & 1.334 & 1.349 \\
\cline { 2 - 7 } & 1 & 1.350 & 1.368 & 1.359 & 1.344 & 1.435 & 1.881 \\
\hline \multirow{3}{*}{$\mathrm{TM}_{21}$} & 0 & 1.879 & 1.927 & 1.876 & 1.881 & 1.934 & 2.130 \\
\cline { 2 - 7 } & 0.5 & 2.136 & 2.167 & 2.128 & 2.119 & 2.203 & 2.239 \\
\cline { 2 - 7 } & 1 & 2.256 & 2.280 & 2.258 & 2.235 & 2.353 & 2.588 \\
\hline \multirow{3}{*}{$\mathrm{TM}_{31}$} & 0 & 2.596 & 2.665 & 2.584 & 2.594 & 2.356 & 2.927 \\
\cline { 2 - 7 } & 0.5 & 2.951 & 2.994 & 2.930 & 2.921 & 2.645 & 3.080 \\
\cline { 2 - 7 } & 1 & 3.106 & 3.150 & 3.109 & 3.080 & 2.829 & \\
\hline
\end{tabular}


The unknown coefficient values of the model given by (6) are optimized by the genetic optimization algorithm just described. The optimum values found are

$$
\beta_{1}=0.12, \quad \beta_{2}=2.54, \beta_{3}=3.65, \beta_{4}=0.23
$$

The effective patch radius expression, $a_{\text {eff }}$, is obtained by substituting the coefficient values given by (11) into (6).

\section{IV.Results and Discussion}

In order to determine the most appropriate suggestion given in the literature, we compared our computed values of the resonant frequencies for the fundamental mode of circular microstrip antenna with the theoretical and experimental results reported by other scientists [22, 23, 26, and 27], all of which are given in Table I.

In order to check the accuracy of the model for two-layered case, the results are compared with an experimental and theoretical values presented in the previous work $[12,13,16$, 17, and 30] in Table II.

Figure.3 show the resonant frequency against the air gap thickness for several radius values of the circular-disc patch. It is seen that the operating frequency increases with the air layer thickness for a given value of patch size. So, antenna tuning is possible by introducing the air gap without changing the antenna parameters.

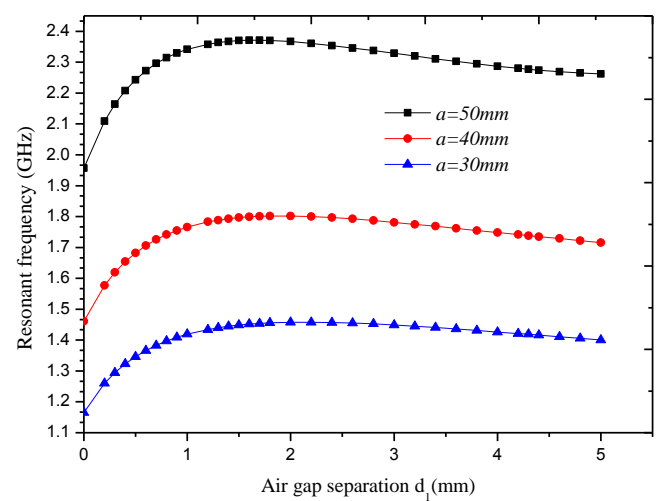

Fig .3. Resonant frequency versus air gap thickness for different values of patch radius, $d_{2}=1.27 \mathrm{~mm}$, and $\varepsilon_{x}=\varepsilon_{z}=2.32$.

It is observed that when the air separation grows, the resonant frequency increases rapidly until achieving a maximum operating frequency at a definite air separation $d_{\text {lfmax. }}$ Note that the effect of the air gap is more pronounced for small values of $d_{l}$ show "Figure. 3". When the air separation exceeds d1fmax, increasing the air gap width will decrease slowly the resonant frequency. These behaviors agree with those discovered theoretically for resonant frequency of circular patch antenna [16-19]. however, it depends inversely on the patch size for a given air gap width $d_{l}$.

Next, the effect of uniaxial anisotropy on the resonant frequency is analyzed.

Fig. 4 depicts the influence of the patch radius on the resonant frequency of a circular microstrip antenna for anisotropic dielectric substrates (without air gap): Boron nitride $\left(\varepsilon_{x}=5.12, \varepsilon_{z}=3.4\right)$. The substrate has thickness $d=1.27 \mathrm{~mm}$. As it can be seen, the resonant frequencies reduce considerably with the dielectric substrates of Boron nitride.

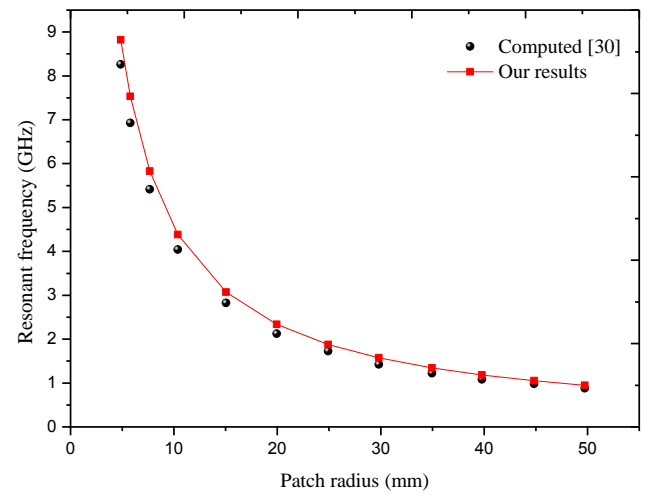

Fig. 4. Resonance frequency as a function of radius patch of a circular microstrip antenna withot air gap on anisotropic substrate, $\left(\varepsilon_{x}=5.12, \varepsilon_{z}=3.4\right)$, $d=1.27 \mathrm{~mm}$.

Also it observed that the resonant frequency increases with the patch radius.

Fig. 5 depict the influence of the air gap thickness on the resonant frequency of a circular-disc microstrip patch for three anisotropic dielectric substrates: Boron nitride $\left(\varepsilon_{z}=3.4, \varepsilon_{x}=5.12\right), \quad$ Epsilam-10 $\quad\left(\varepsilon_{z}=10.3, \varepsilon_{x}=13\right)$, and Sapphire $\left(\varepsilon_{z}=11.6, \varepsilon_{x}=9.4\right)$. The substrate has thickness $d_{l}=$ $1.27 \mathrm{~mm}$ and the air gap width is varied from $0 \mathrm{~mm}$ to $5 \mathrm{~mm}$.

As it can be seen, the resonant frequency reduces considerably when the dielectric substrate changes from Boron nitride to Epsilam-10, and this is in contrast to what happens when the medium changes from Epsilam-10 to Sapphire. The obtained results show that when the permittivity $\varepsilon_{z}$ is changed and $\varepsilon_{x}$ remains constant, the resonant frequency changes drastically, on the other hand, we found a slight shift in the resonant frequency 
doi:10.15849/icit.2015.0117 @ ICIT 2015 (http://icit.zuj.edu.jo/ICIT15)

when the permittivity $\varepsilon_{x}$ is changed and $\varepsilon_{z}$ remains constant.

These behaviors agree very well with those reported by [6]. Also it is observed that the resonant frequency increases with the air gap thickness.

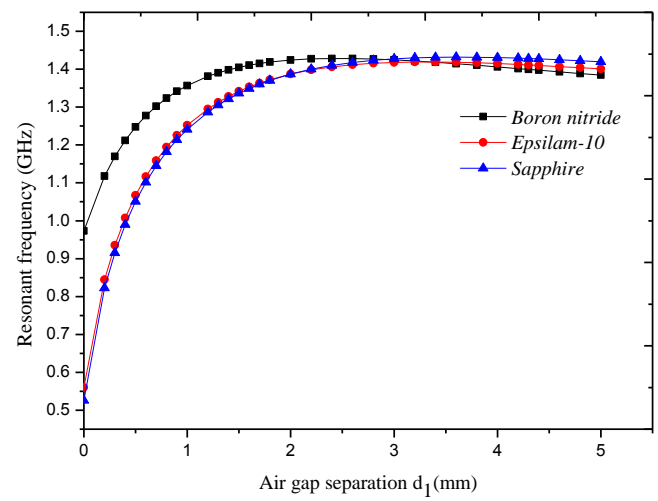

Fig. 5. Resonant frequency versus air gap thickness for different anisotropic dielectric substrates.

\section{V.Conclusion}

In this paper, a simple CAD formulation is presented based the cavity model for simple circular disc microstrip antenna is extended with some modifications for the tunable geometry taking into account the anisotropy in the layer. Computations show that the air separation can be adjusted to have the maximum operating frequency of the antenna. Extreme care should be taken when designing a microstrip antenna with thin air gap; since small uncertainty in adjusting the air separation can result in an important detuning of the frequency. The effects of a uniaxial substrate on the resonant frequency of structures are considered in detail. The results of the study will also be useful in the microstrip disk antenna design using uniaxial metamaterials.

\section{References}

[1] Z.-S. Duan, S.-B. Qu, Y. Wu and J.-Q. Zhang, "Wide bandwidth and broad beamwidth microstrip patch antenna", Electron. Lett., Vol. 45 No. 5, 2009.

[2] N. G. Alexopoulos, "Integrated circuit structures on anisotropic substrates," IEEE Trans. Microwave Theory Tech., vol. MTT-33, pp. 847-881, 1985 .

[3] N. G. Alexopoulos and S. A. Maas, "Characteristics of microstrip directional couplers on anisotropic substrates," IEEE Trans. Microwave Theory Tech., vol. MTT-30, pp. 1267-1270, 1982.

[4] E. Drake, R. R. Boix, M. Horno, and T. K. Sarkar, "effect of substrate dielectric anisotropy on the frequency behavior of microstripcircuits", IEEE Trans. Microwave Theory Tech., vol. MTT-30, pp. 1267-1270, 1982.

[5] H. Y. Yang and N. G. Alexopoulos, "Uniaxial and biaxial substrate effects on finline characteristics," IEEE Trans. Microwave Theory Tech., vol. MTT- 35, pp. 24-29, 1987.
[6] F. Bouttout, F. Benabdelaziz, A. Benghalia, D. Khedrouche and T. Fortaki "Uniaxially anisotropic substrate effects on resonance of rectangular microstrip patch antenna" Electron. Lett., vol. 35, No 4, pp. 255-256, 1999.

[7] A. Zhao, J. Juntunen, and A. V. Raisanen, "An Efficient FDTD Algorithm for the Analysis of Microstrip Patch Antennas Printed on a

General Anisotropic Dielectric Substrate," IEEE Trans. Microwave Theory Tech, vol. 47, NO. 7, 1999.

[8] A. Luiz P. S. Campos and A. G. d'Assunqiio "Hertz Vector Potential Analysis of FSS on Anisotropic Substrates" Proceedings SBMO/IEEE MTT-S IMOC 2003.

[9] V. Losada, Boix R R, and Horno M. Full-wave analysis of circular microstrip resonators in multilayered media containing uniaxial anisotropic dielectrics, magnetized ferrites, and chiral materials," IEEE Trans. Microwave Theory Techniques, Vol. 48, $1057-1064,2000$.

[10] C. S. Gurel, and Yazgan E. Characteristics of a circular patch microstrip antenna on uniaxially anisotropic substrate. IEEE Trans. Antennas Propagat, Vol. 52; 2532-2537, 2004.

[11] K.F. Lee, K.Y. Ho, and J.S. Dahele, "Circular disk microstrip 1984. antenna with an air gap", IEEE Trans Antennas Propag 32, 880- 884 ,

[12] J.S. Dahele and K.F. Lee, "Theory and experiment on microstrip antennas with air gap", Proc IEE 132 (Part H), 455-460, 1985.

[13] F. Abboud, J.P. Damiano, and A. Papiernik, "A new model for calculating the input impedance of coax-fed circular microstrip antennas with and without air gap", IEEE Trans Antennas Propag 38, 1882-1885, 1990.

[14] J.S. Joy and B. Jecko, "A formula for the resonance frequencies of circular microstrip patch antenna satisfying CAD requirements", Int J RF

Microwave Comp Aided Eng., Vol. 3, 67-70, 1993.

[15] K. Guney, "Resonant frequency of electrically-thick circular microstrip antenna", Int J Electron., Vol. 77 , 377-385, 1994.

[16] C, .S. Gurel and E. Yazgan, "Resonant frequency of an air gap tuned circular disk microstrip antenna", Int J Electron., Vol. 87, 973-979, 2000.

[17] D. Guha, "Resonant frequency of circular microstrip antennas with and without air gaps", IEEE Trans Antennas Propag., Vol. 49, 5559 , 2001.

[18] T. Gunel, "Continuous hybrid approach to the modified resonant frequency calculation for circular microstrip antennas with and without air gaps", Microwave Opt Tech Lett., Vol. 40, 423- 427, 2004.

[19] C .S. Gurel, E. Aydın, and E. Yazgan, "Modified resonant frequency calculation for two-layered circular patch microstrip antenna", Microwave Opt Tech Lett., Vol. 49 , 2263-2267, 2007.

[20] H.I. Kang and J.T. Song, "Electrically tunable rectangular microstrip antenna", Electron. Lett., Vol. 146 No 7, 2010.

[21] Y. Tighilt, F. Bouttout, and A. Khellaf, "Modeling and Design of Printed Antennas Using Neural Networks", Int J RF and Microwave CAE., Vol. 21:228-233, 2011.

[22] T. Itoh and R. Mittra, Analysis of a microstrip disk resonator, AEU Int J Electron Commun., Vol. 27, 456-458, 1973.

[23] J.Q. Howell, Microstrip antennas, IEEE Trans Antennas Propagat AP23, 90-93, 1975.

[24] S.A. Long, L.C. Shen, M.D. Walton, and M.R. Allerding, Impedance of a circular disk printed- circuit antenna, Electron Lett., Vol. 14, 684- 686, 1978. 


\section{ICIT 2015 The $7^{\text {th }}$ International Conference on Information Technology}

doi:10.15849/icit.2015.0117 @ ICIT 2015 (http://icit.zuj.edu.jo/ICIT15)

[25] S. Yano and A. Ishimaru, A theoretical study of the input impedance of a circular microstrip disk antenna, IEEE Trans Antennas Propagat AP- 29, 77-83, 1981.

[26] F. Abboud, J.P. Damiano, and A. Papiernik, "New determination of resonant frequency of circular disc microstrip antenna: application to thick substrate", Electron Lett., Vol. 24, 1104-1106, 1988.

[27] Q. Liu and W.C. Chew, "Curve-fitting formulas for fast determination of accurate resonant frequency of circular microstrip patches", IEE Proc Microwave Antennas Propagat PtH 135, $289-$ $292,1988$.

[28] Anirban Karmakar , Rowdra Ghatak , R.K. Mishra , D.R. Poddar,

"Sierpinski carpet fractal-based planar array optimization based on differential

evolution algorithm", Journal of Electromagnetic Waves and Applications., Vol 29, 247-260, 2015

[29] Sharmin Shabnam, Suvrajit Manna, Udit Sharma, Pinaki Mukherjee., "Optimization of Ultra Wide-Band Printed Monopole Square Antenna Using Differential Evolution Algorithm", Advances in Intelligent Systems and Computing Vol. 339, pp 81-89, 2015

[30] A. K. Verma, and Nasimuddin, "Analysis of circular microstrip patch antenna as an equivalent rectangular microstrip patch antenna on iso/anisotropic thick substrate," IEE proc. Microw. Antennas propag. vol.150, No. 4, pp. 223-229, 2003.

[31] HFSS: High Frequency Structure Simulator-Ansoft Corp. 